\title{
Generalized Cross-Layer Designs for Generic Half-Duplex Multicarrier Wireless Networks with Frequency-Reuse
}

\author{
Rozita Rashtchi, Student Member, IEEE, Ramy H. Gohary, Senior Member, IEEE, and Halim Yanikomeroglu, \\ Senior Member, IEEE
}

\begin{abstract}
In this paper, joint designs of data routes and resource allocations are developed for generic half-duplex multicarrier wireless networks in which each subcarrier can be reused by multiple links. Two instances are considered. The first instance pertains to the general case in which each subcarrier can be timeshared by multiple links, whereas the second instance pertains to a special case in which time-sharing is not allowed and a subcarrier, once assigned to a set of links, is used by those links throughout the signalling interval. Novel frameworks are developed to optimize the joint design of data routes, subcarrier schedules and power allocations. These design problems are nonconvex and hence difficult to solve. To circumvent this difficulty, efficient techniques based on geometric programming are developed to obtain locally optimal solutions. Numerical results show that the designs developed in both instances yield performance that is superior to that of their counterparts in which frequency-reuse is not allowed.
\end{abstract}

Index Terms-Power control, geometric programming, monomial approximation, time-sharing, self-concordance.

\section{INTRODUCTION}

The prospect of having ubiquitous high data-rate wireless services is leveraged by the versatility and portability of the communication devices that will form the nodes of future wireless networks. These devices will be able to perform various functions including sending, receiving and/or relaying data to other nodes. As such, it is expected that future wireless networks will not possess a predetermined topology, but rather an $a d$ hoc one that encompasses many existing and upcoming network structures including current and relay-aided cellular networks [1], [2].

Given the stringent limitations on the spectrum available for wireless communications, providing high data-rate services banks on sharing the spectrum by multiple users which results in potentially significant interference. One option to mitigate interference is to use the Orthogonal Frequency Division Multiple Access (OFDMA) technique, wherein a set of orthogonal narrow-band subcarriers are exclusively assigned to

The authors are with the Department of Systems and Computer Engineering, Carleton University, Ottawa, Ontario, Canada, \{rozita, gohary, halim\}@sce.carleton.ca

Preliminary versions of this work were presented, in part, at the IEEE Glob. Telecommun. (Globecom), 2013, and, in part, at the IEEE Int. Wkshp. Signal Processing Advances in Wireless Commun. (SPAWC), 2014.

This work is supported in part by a Discovery Grant (DG) of the Natural Sciences and Engineering Research Council (NSERC) of Canada, in part by Huawei Canada Co., Ltd., and in part by the Ontario Ministry of Economic Development and Innovations ORF-RE (Ontario Research Fund - Research Excellence) program. each user. This technique offers several advantages including design simplicity and resilience to frequency-selective fading. In spite of these advantages, rate-effective utilization of the available spectrum may require the OFDMA subcarriers to be used simultaneously, rather than exclusively, by multiple users. This is especially the case when the network is composed of essentially separated clusters. In contrast, for tightly coupled networks exclusive usage of subcarriers can be more beneficial from a rate perspective [3], [4].

In order for a wireless network to be able to support the reliable communication of high data rates, the scarce resources available for the network must be carefully exploited. Such resources include the spectrum available for communication, time, and the typically low power of the wireless nodes. Proper exploitation of these resources involves choosing the optimal routes of the data flows, the optimal powers to be allocated by the nodes to each subcarrier, and the optimal scheduling and possibly duration over which the subcarriers are assigned to various links. Although these tasks have traditionally been performed separately, they are interrelated and performing them in isolation may incur a significant loss in performance.

To avoid the aforementioned drawback, we devise a joint optimization framework that incorporates data routing, subcarrier scheduling and power allocation in the design of a generic multicarrier network. The network is generic in the sense that it possesses an ad hoc topology and its nodes can assume multiple roles simultaneously including being sources, destinations and/or relays. This framework is centralized, in the sense that the design is performed by a central entity that is aware of the network parameters. Hence, this framework can be seen as a benchmark for distributed and potentially less comprehensive designs. In this framework each subcarrier can be reused by multiple links, and the nodes acting as relays operate in the half-duplex mode, i.e., a node cannot send and receive at the same time on the same subcarrier. The objective of the design is to maximize a weighted-sum of the rates injected and reliably communicated over the network. Weights are assumed to be known a priori, but can be adapted over time to account for fairness issues and to maintain a desired quality of service.

Two instances of networks are considered. In the first instance, each subcarrier can be time-shared by multiple links, thereby resulting in continuous subcarrier scheduling variables. The role of these variables is to determine the fraction of time during which a subcarrier is used over a particular link. In 
contrast with the first instance, in the second one time-sharing is not allowed and a subcarrier, once assigned to a set of links, is used by those links throughout the signalling interval. This instance results in binary subcarrier schedules, which using a particular change of variables, are incorporated in the power allocation constraints. This results in a design problem that is significantly easier than its general counterpart considered in the first instance. It is worth noting that the first instance is a generalization not only of the second instance, but also of instances in which frequency-reuse is not permitted [5]. As such, the framework considered in this instance offers significant performance advantages over currently available designs, but at the expense of increasing dimensionality and design complexity. This instance provides an inherent tradeoff between performance and design complexity; the design complexity can be reduced by restricting the number of links that can reuse a particular subcarrier.

The optimization problems arising from the joint design in both instances are nonconvex and hence difficult to solve. To overcome this difficulty, a logarithmic transformation is used to cast the original problem in a form that, for all but a few constraints, complies with the geometric programming (GP) standard form [6]. The constraints that are not compatible with that form are approximated by monomial expressions that correspond to their first order Taylor expansion around a given initial point [7]. Using an exponential transformation, the resulting approximation can be cast in a convex form. A refinement of this approximation can be obtained by iterative updating of the initial point. In particular, we use the socalled iterative monomial approximation technique, wherein the solution of one convex approximation is used as the initial point in the following iteration. Under relatively mild conditions, this technique is guaranteed to yield a solution of the Karush-Kuhn-Tucker (KKT) system corresponding to the original problem [8]. Numerical results show that the designs developed in both instances yield performance that is considerably superior to that of their counterparts in which frequency-reuse is not allowed.

In comparison with currently available designs, the ones presented herein are the first to attempt designing data routes, subcarrier schedules and power allocation jointly when the subcarriers are both time-shared and frequency-reused. In particular, the contributions in this paper include: 1) introducing the concept of simultaneous time-sharing and frequency-reuse of subcarriers; 2) casting the joint design of data routes, subcarrier schedules and power allocation in a framework that is amenable to GP-based optimization; 3) providing a simplified approach that enables mustering a considerable portion of the gains offered by the full joint design, but with a significantly lower complexity; and 4) developing an efficient polynomial complexity algorithm for the special case in which the subcarriers can be frequency-reused but not time-shared. This work builds on the results obtained in [9] and [10]. However, the exposition herein is more comprehensive and includes additional examples, a simplified approach and complexity analysis.

The paper is organized as follows. Section $\amalg$ provides an overview of currently available design techniques. Section III explains the system model and design objective. Section IV considers the joint design of data routes and power allocations when time-sharing of subcarriers is allowed. The complementary instance in which time-sharing is not allowed is addressed in Section $\mathrm{V}$. The complexity of the proposed algorithms are examined in Section VI Numerical results are provided in Section VII and Section VIII concludes the paper. For completeness, the GP standard form and the monomial approximation technique are provided in Appendix $\mathrm{A}$ and the derivation of the results pertaining to complexity is provided in Appendix B.

\section{RELATED WORK}

In this section we provide an overview of the currently available techniques for routing and resource allocation in wireless networks. A plethora of techniques is available for optimizing each aspect in isolation, but significantly fewer ones consider their optimization jointly.

Resource allocation in wireless networks constitutes the task of determining the power allocated for each transmission and the fraction of time over which a particular subcarrier is assigned to that transmission. Instances in which resource allocation techniques were developed are provided in [6], [11]-[15] for various network scenarios. For instance, power allocation techniques for single-carrier cellular systems and ad hoc multicarrier systems were developed in [11] and [6], respectively. To enable more effective utilization of resources, power allocations were optimized jointly with binary-constrained subcarrier schedules. For instance, the designs developed in [12] and [13] rely on the premise that each subcarrier is exclusively used by one node and the solutions obtained therein are potentially suboptimal. When the binary constraint on the subcarrier schedules is relaxed allowing the subcarriers to be time-shared by multiple nodes, the optimal power allocations can be shown to be the waterfilling ones [14]; a related problem was considered in [15] for a case in which the nodes experience self-noise.

Further improvement can be achieved by joint optimization of resource allocations and routing [5], [16]-[19]. For instance, a method for obtaining jointly optimal routes and power allocations was developed in [16] for the case in which the nodes were restricted to use orthogonal channels for their transmissions. In a complementary fashion, the case in which the power allocations are fixed was considered in [17]. Therein, a heuristic was developed for optimizing the data routes and subcarrier schedules jointly.

Capitalizing on the potential gains of incorporating power allocation jointly with data routing and subcarrier scheduling, the authors considered a generic network in which the nodes can assume multiple roles at the same time and each subcarrier could be either used exclusively by one link or time-shared by multiple links [5]. Although the designs provided in [5] offer an effective means for exploiting the resources available for the network, these designs restrict the subcarriers to be used exclusively by only one link at any given time instant. Such a restriction may not incur a significant performance loss in tightly coupled networks [3], but in networks with 
clustered structures, this restriction can be quite harmful. For unclustered networks, frequency-reuse may result in a substantial increase in the interference levels. However, if properly exploited, frequency-reuse can yield valuable performance gains. The effect of frequency-reuse was considered in singlechannel networks in [18] for the case in which the data rates are restricted to assume discrete values, and in [19] for the case in which the nodes use superposition coding.

In the current work, we will consider the joint optimization of power allocations, subcarrier schedules and data routes in the design of generic multicarrier networks with frequencyreuse, and with and without time-sharing. As such, these designs generalize currently available ones, and will subsequently offer a significant improvement over their performance.

A summary of this review and a comparison to our work is presented in Table [

\section{Problem Statement}

\section{A. System Model}

We consider a multicarrier wireless network of $N$ nodes, each with one transmit and one receive antenna, and a fixed power budget, $P_{n}, n \in \mathcal{N} \triangleq\{1,2, \cdots, N\}$. The network operates over a frequency-selective broadband channel of bandwidth $W_{0}$, which is partitioned into $K$ frequency-flat narrowband channels, each of bandwidth $W=\frac{W_{0}}{K}$. Node are assumed to be capable of simultaneously transmitting, receiving and relaying data. This assumption is generic, in the sense that constraining some nodes to perform a subset of tasks can be readily incorporated in the formulations that will be developed hereinafter. For tractability, the nodes are assumed to always have data ready for transmission [16], and for practical considerations, the relaying nodes are assumed to operate in a multi-hop, rather than cooperative, half-duplex mode [17.

The nodes are connected with $L$ wireless links, each composed of $K$ subcarriers and the set of all links is denoted by $\mathcal{L} \triangleq\{1,2, \cdots, L\}$. The coefficient of the $k$-th subcarrier of link $\ell$ connecting node $n$ to node $n^{\prime}$ is denoted by the complex number $h_{n n^{\prime}}^{(k)}$ which comprises pathloss, shadowing and fading. An instance of such a network with $N=4$ nodes and $K=2$ subcarriers is depicted in Figure 1 .

In addition to their desired signals, the nodes receive a superposition of noise and interference due to the transmissions of other nodes in the network. Denoting the signals transmitted to and received by node $n$ on the $k$-th subcarrier by $u_{n}^{(k)}$ and $y_{n}^{(k)}$, respectively, we can write $y_{n^{\prime}}^{(k)}=\sum_{n \in \mathcal{N} \backslash\left\{n^{\prime}\right\}} h_{n n^{\prime}}^{(k)} u_{n}^{(k)}+$ $v_{n^{\prime}}^{(k)}$, where ' $\backslash$ ' denotes the set-minus operation and $v_{n^{\prime}}^{(k)}$ denotes the corresponding zero-mean additive Gaussian noise with variance $N_{0}$. Assuming, as before, that $\ell \in \mathcal{L}$ is the link connecting node $n$ to node $n^{\prime}$, it can be seen that the signalto-noise-plus-interference ratio (SNIR) observed by node $n^{\prime}$ on subcarrier $k$ of link $\ell$ is given by

$$
\operatorname{SNIR}(\ell, k)=\frac{p_{n k}\left|h_{n n^{\prime}}^{(k)}\right|^{2}}{W N_{0}+\sum_{n^{\prime \prime} \in \mathcal{N} \backslash\left\{n, n^{\prime}\right\}} p_{n^{\prime \prime}}^{(k)}\left|h_{n^{\prime \prime} n^{\prime}}^{(k)}\right|^{2}},
$$

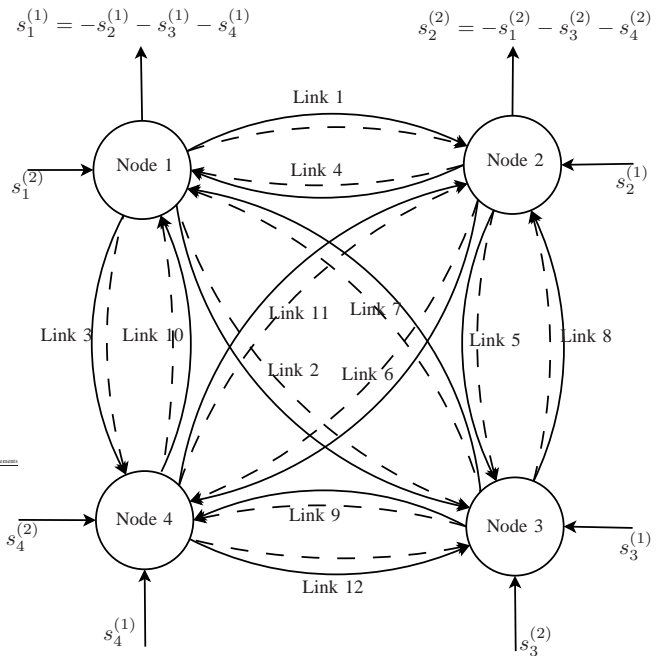

Fig. 1: An exemplary network with $N=4, K=2$ and $D=\{1,2\}$. The objective is to maximize a weighted sum of $\left\{s_{n}^{(d)}\right\}$, i.e., the rate injected at node $n$ and intended for destination $d$.

where $p_{n k}$ is the power allocated by node $n$ to the $k$-th subcarrier. The second term in the denominator of (1) represents the aggregate interference observed by node $n^{\prime}$ on subcarrier $k$ of link $\ell$. When the nodes transmit Gaussian distributed signals, the maximum data rate that can be reliably communicated on this subcarrier is given by $W \log _{2}(1+\operatorname{SNIR}(\ell, k))$.

For ease of exposition, we divide both the numerator and denominator of (1) by $W N_{0}$ and we use $g_{\ell k}$ to denote the normalized channel gain, $\frac{\left|h_{n n}^{(k)}\right|^{2}}{W N_{0}}$, between any two nodes $n, n^{\prime} \in \mathcal{N}$.

\section{B. Network Topology}

The considered network can be represented by a fullyconnected weighted directed graph with $N$ vertices and $L=$ $N(N-1)$ links. To facilitate enumeration of links, the link from node $n$ to node $n^{\prime}$ will be labelled by $\ell=(N-1)(n-$ 1) $+n^{\prime}-1$ if $n<n^{\prime}$ and by $\ell=(N-1)(n-1)+n^{\prime}$ if $n>n^{\prime}$. The sets of incoming and outgoing links of node $n \in \mathcal{N}$ are denoted by $\mathcal{L}_{-}(n)$ and $\mathcal{L}_{+}(n)$, respectively, and the connectivity of this graph can be captured by an incidence matrix, $A=\left[a_{n \ell}\right]$, where $a_{n \ell}=1$ if $\ell \in \mathcal{L}_{+}(n), a_{n \ell}=-1$ if $\ell \in \mathcal{L}_{-}(n)$ and $a_{n \ell}=0$ otherwise [16].

\section{Design Objective}

Let $\mathcal{D} \triangleq\{1, \ldots, D\}$ be the set of all destination nodes, where $\mathcal{D} \subseteq \mathcal{N}$. Let $s_{n}^{(d)}$ be the rate of the data stream injected into node $n \in \mathcal{N}$ and intended for destination $d \in \mathcal{D}$. The objective of our joint design is to maximize a weighted-sum of the rates injected into the network, i.e., $\max \sum_{d \in \mathcal{D}} \sum_{n \in \mathcal{N} \backslash\{d\}} w_{n}^{(d)} s_{n}^{(d)}$, where $\left\{w_{n}^{(d)}\right\}$ are nonnegative weights satisfying $\frac{1}{D(N-1)} \sum_{d \in \mathcal{D}} \sum_{n \in \mathcal{N} \backslash\{d\}} w_{n}^{(d)}=$ 1.

Assigning weights to the injected rates provides a convenient means for controlling the quality of service (QoS); a higher weight implies a higher priority. Weights are typically 
TABLE I: Related Work

\begin{tabular}{|c|c|c|c|c|c|}
\hline Platform & Routing & Power Allocation & Scheduling & Frequency-reuse & Reference \\
\hline Multiuser system & $\times$ & $\checkmark$ & $\checkmark$ & $\checkmark$ & 3 \\
\hline Uplink cellular & $x$ & $\checkmark$ & $\bar{x}$ & $\checkmark$ & $6], 11]$ \\
\hline OFDMA & $x$ & $\checkmark$ & $\checkmark$ & $x$ & [12], [13], [14], [15] \\
\hline Generic network & $\sqrt{ }$ & $\sqrt{ }$ & $x$ & $x$ & 16 \\
\hline Mesh network & $\checkmark$ & $x$ & $\checkmark$ & $x$ & 17 \\
\hline Generic network & $\checkmark$ & $\sqrt{ }$ & $x$ & $\sqrt{ }$ & [18, [19] \\
\hline Generic network & $\checkmark$ & $\checkmark$ & $\bar{\checkmark}$ & $x$ & 5 \\
\hline Generic network & $\sqrt{ }$ & $\sqrt{ }$ & $\sqrt{ }$ & $\checkmark$ & This work \\
\hline
\end{tabular}

assigned a priori, but can be adapted to meet QoS requirements [15]. Varying the weights enables us to determine the set of all rates that the proposed design can simultaneously achieve.

Having described the system model, in Section IV we will characterize the constraints that must be satisfied by the routes, the subcarrier schedules, the data rates and the power allocations.

\section{General Case: Routing and Resource ALLOCATION WITH TIME-SHARING}

We consider the case when each subcarrier can be both reused and time-shared by multiple links. This case generalizes the cases in which either frequency-reuse or time-sharing of subcarriers is not considered, e.g., [5]. After characterizing the constraints that must be satisfied by the network variables, we will formulate the cross-layer design as an optimization problem. Unfortunately this problem is nonconvex and to obtain a solution of its KKT system, we will use an iterative GP-based technique that is guaranteed to converge to such a solution.

\section{A. System Constraints}

In this section, we derive the mathematical constraints that must be satisfied by any feasible set of data routes, timesharing schedules and power allocations.

1) Routing Constraints: Let $x_{\ell k}^{(d)}$ be the data flow intended for destination $d \in \mathcal{D}$ on subcarrier $k \in \mathcal{K}$ of link $\ell \in \mathcal{L}$. The flows, $\left\{x_{\ell k}^{(d)}\right\}$, and the injected rates, $\left\{s_{n}^{(d)}\right\}$, are related by the flow conservation law, which must be satisfied at each node. This law stipulates that the sum of flows intended for any destination $d \in \mathcal{D}$ at each node must be equal to zero [16]. Applying this law to the current network and using the incidence matrix in Section $\amalg$ II-B it can be seen that $\left\{x_{\ell k}^{(d)}\right\}$ and $\left\{s_{n}^{(d)}\right\}$ must satisfy the following constraints:

$$
\sum_{\ell \in \mathcal{L}} \sum_{k \in \mathcal{K}} a_{n l} x_{\ell k}^{(d)}=s_{n}^{(d)}, \quad n \in \mathcal{N} \backslash\{d\}, d \in \mathcal{D} .
$$

The flow conservation law implies that the rate of data leaving the network at $d \in \mathcal{D}$ equals the sum of the data rates injected into the network and intended for this destination. Hence, we can write $s_{d}^{(d)}=-\sum_{n \in \mathcal{N} \backslash\{d\}} s_{n}^{(d)}$. The injected rates, $\left\{s_{n}^{(d)}\right\}_{n \neq d}$, are non-negative, and since the network is represented by a directed graph, the flows, $\left\{x_{\ell k}^{(d)}\right\}$, must be also non-negative. Hence,

$$
s_{n}^{(d)} \geq 0, \quad n \in \mathcal{N} \backslash\{d\}, d \in \mathcal{D},
$$

$$
x_{\ell k}^{(d)} \geq 0, \quad \ell \in \mathcal{L}, k \in \mathcal{K}, d \in \mathcal{D} .
$$

2) Scheduling Constraints: Considering both time-sharing and frequency-reuse requires introducing a set of variables to characterize the fraction of time over which a particular subset of links utilize the same subcarrier. To do so, let $\gamma_{\ell_{1} \ldots \ell_{m}}^{(k)}$ be the fraction of the signalling interval during which links $\ell_{1}, \ldots, \ell_{m} \in \mathcal{L}$ are simultaneously 'active' on subcarrier $k \in \mathcal{K}$; the remaining $L-m$ links in $\mathcal{L}$ are 'silent' on this subcarrier. Without loss of generality, we will write the indices in an ascending order, i.e., $\ell_{1}<\cdots<\ell_{m}$. For notational convenience, let $\Gamma$ be the set of all the subcarrier time-sharing schedules. The cardinality of $\Gamma$ is given by $|\Gamma|=K \sum_{i=1}^{L}\left(\begin{array}{c}L \\ i\end{array}\right)=K\left(2^{L}-1\right)$. For instance, consider a network with $L=3$ links and $K=1$ subcarriers. In this case, $\Gamma=\left\{\gamma_{1}^{(1)}, \gamma_{2}^{(1)}, \gamma_{3}^{(1)}, \gamma_{1,2}^{(1)}, \gamma_{1,3}^{(1)}, \gamma_{2,3}^{(1)}, \gamma_{1,2,3}^{(1)}\right\}$ and $|\Gamma|=7$. To see the role of $\Gamma$, consider the schedules in Figure 2 In this figure, $\gamma_{1}^{(1)}=0.5, \gamma_{1,2}^{(1)}=0.2, \gamma_{1,2,3}^{(1)}=0.3$, and all the other elements in $\Gamma$ are zero.

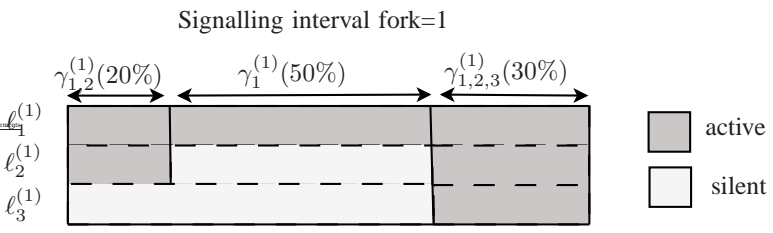

Fig. 2: An exemplary scheduling table for a network with $L=3$, $K=1$.

Note that the fact that the channels are assumed constant over the signalling interval implies that only the time-sharing schedules (i.e., entries of $\Gamma$ ) affect the rate expressions, irrespective of the particular time interval over which the subcarriers are time-shared. In other words, horizontal displacement of the shaded blocks in Figure 2 does not affect the rate expressions.

The number of variables in $\Gamma$ grows exponentially with the number of links, $L$. This renders the incorporation of $\Gamma$ in the joint optimization computationally prohibitive. In most cases this complexity can be significantly reduced without incurring heavy performance losses. For instance, if the network is tightly coupled, high interference levels render the reuse of subcarriers on multiple links less beneficial. In such a case, restricting the reuse of a subcarrier to a fewer links may incur negligible deterioration in performance but reduces the number of variables significantly. To take advantage of this observation, we limit the number of links that can reuse a particular subcarrier to $I \ll L$. By performing this restriction, 
the number of elements in $\Gamma$ is reduced from $K\left(2^{L}-1\right)$ to $K \sum_{i=1}^{I}\left(\begin{array}{c}L \\ i\end{array}\right)$, which, for small $I$, is polynomial in $L$. For instance, if at most two links are allowed to reuse a particular subcarrier at any given time, i.e., $I=2$, the number of elements in $\Gamma$ reduces to $\frac{L(L+1)}{2}$. It is worth noting that limiting the number of simultaneous transmissions, $I$, inherently offers a trade-off between the performance and complexity. In particular, as $I$ increases, the available resources are utilized more efficiently. However, our simulations suggest that most of the gain of time-sharing and frequency-reuse is accrued by only considering $I \leq 3$ simultaneous transmissions.

For feasible time-sharing schedules, the elements in $\Gamma$ must be non-negative and, to ensure no overlapping in time, the total time over which any subcarrier $k \in \mathcal{K}$ is used must not exceed the length of the signalling interval. These constraints imply that

$$
\begin{array}{lr}
\Gamma \geq 0, & \text { elementwise }, \\
\sum_{m=1}^{I} \sum_{\ell_{1} \cdots \ell_{m} \in \mathcal{L}} \gamma_{\ell_{1} \ldots \ell_{m}}^{(k)} \leq 1, & \forall k \in \mathcal{K} .
\end{array}
$$

Note that summations in (6) characterize the number of links, $m$, that reuse a particular subcarrier $k$. For instance, for the case in which at most two links reuse this subcarrier, the left hand side (LHS) of (6) can be expressed as $\sum_{\ell_{1} \in \mathcal{L}} \gamma_{\ell_{1}}^{(k)}+$ $\sum_{\ell_{1} \in \mathcal{L}} \sum_{\ell_{2} \in \mathcal{L}} \gamma_{\ell_{1} \ell_{2}}^{(k)}$.

Nodes cannot broadcast data to multiple destinations at the same time, that is, at any time instant, node $n$ can have at most one active link on subcarrier $k$. Hence, the time-sharing schedules corresponding to multiple outgoing links of node $n$ must be zero. This can be represented as

$$
\begin{aligned}
a_{n \ell_{1}}^{+} a_{n \ell_{2}}^{+}\left(\gamma_{\ell_{1} \ell_{2}}^{(k)}+\sum_{m=3}^{I} \sum_{\ell_{3} \cdots \ell_{m} \in \mathcal{L}} \gamma_{\ell_{1} \ldots \ell_{m}}^{(k)}\right)=0, \\
\ell_{1} \in \mathcal{L}, \ell_{2} \in \mathcal{L} \backslash\left\{\ell_{1}\right\}, k \in \mathcal{K},
\end{aligned}
$$

where $a_{n \ell}^{+}=\max \left\{0, a_{n \ell}\right\}$, that is, $a_{n \ell}^{+}=1$ if $\ell \in \mathcal{L}_{+}(n)$ and zero, otherwise.

To enforce the half-duplex constraint, we must ensure that no two links, $\ell_{1} \in \mathcal{L}_{-}(n)$ and $\ell_{2} \in \mathcal{L}_{+}(n)$, can be active on the same subcarrier $k \in \mathcal{K}$ at the same time. This implies that all the time-sharing schedules that correspond to $\ell_{1}$ and $\ell_{2}$, i.e., $\gamma_{\ell_{1} \ldots \ell_{m}}^{(k)}, m=2, \ldots, I$, must be zero. Since all the entries in $\Gamma$ are non-negative, these constraints can be written as

$$
\begin{aligned}
a_{n \ell_{1}}^{+} a_{n \ell_{2}}^{-}\left(\gamma_{\ell_{1} \ell_{2}}^{(k)}+\sum_{m=3}^{I} \sum_{\substack{\ell_{3} \cdots \ell_{m} \in \mathcal{L} \\
\ell_{1} \in \mathcal{L}, \ell_{2} \in \mathcal{L} \backslash\left\{\ell_{1}\right\}}} \gamma_{\ell_{1} \ldots \ell_{m}}^{(k)}\right)=0, k \mathcal{K},
\end{aligned}
$$

where $a_{n \ell}^{-}=\left|\min \left\{0, a_{n \ell}\right\}\right|$, that is, $a_{n \ell}^{-}=-1$ if $\ell \in \mathcal{L}_{-}(n)$ and zero, otherwise. Note that (7) and (8) take effect only when $a_{n \ell}^{+} a_{n \ell^{\prime}}^{-} \neq 0$ and $a_{n \ell}^{+} a_{n \ell^{\prime}}^{+} \neq 0$, respectively.

3) Power Allocation Constraints: To facilitate the design, we replace the node power variables $\left\{p_{n k}\right\}$ with link power variables $\left\{q_{\ell k}\right\}$, which are related by the following transformation:

$$
p_{n k}=\max _{\ell \in \mathcal{L}_{+}(n)} q_{\ell k}, \quad n \in \mathcal{N}, k \in \mathcal{K} .
$$

To gain a better understanding of the transformation in (9), we note that (7) implies that, of all the links in $\mathcal{L}_{+}(n)$, only one element in the set $\left\{q_{\ell k}\right\}_{\ell \in \mathcal{L}_{+}(n)}, \forall n \in \mathcal{N}, k \in \mathcal{K}$, can assume a strictly positive value. Now, (9) indicates that this value is the power allocated by node $n$ to subcarrier $k$. Using (9), we will formulate our design in terms of $\left\{q_{\ell k}\right\}$ instead of $\left\{p_{n k}\right\}$. These variables must satisfy the following non-negativity constraints:

$$
q_{\ell k} \geq 0, \quad \ell \in \mathcal{L}, k \in \mathcal{K} .
$$

In a practical network, the nodes are likely to have individual power budgets which bounds the total power used by each node on all subcarriers. To capture this constraint, we note that only the subcarriers scheduled to outgoing links contribute to the power consumption of each node. More specifically, if $\ell_{1} \in \mathcal{L}_{+}(n)$, then all the time-sharing schedules that correspond to $\ell_{1}$ contribute to the power consumption at node $n$. This constraint can be written as

$$
\sum_{k \in \mathcal{K}} \sum_{\ell_{1} \in \mathcal{L}_{+}(n)} q_{\ell_{1} k}\left(\gamma_{\ell_{1}}^{(k)}+\sum_{m=2}^{I} \sum_{\ell_{2} \ldots \ell_{m} \in \mathcal{L}} \gamma_{\ell_{1} \ldots \ell_{m}}^{(k)}\right) \leq P_{n},
$$

4) Capacity Constraints: To complete the characterization of the network, we point out that the data flows and the power allocations are coupled by the maximum aggregate rate that can be supported by the subcarriers of each link. In particular, the aggregate rate $\sum_{d \in \mathcal{D}} x_{\ell k}^{(d)}$ must not exceed the capacity of the $k$-th subcarrier of link $\ell$.

To characterize the capacity constraints, we note that the transmission on link $\ell \in \mathcal{L}$ and subcarrier $k \in \mathcal{K}$ is composed of two parts. The first part accounts for the fraction of time over which this transmission is interference-free, whereas the second part accounts for the fraction of time over which this transmission interferes with other transmissions. To characterize the second part, we identify the interfering links and the fraction of time over which these links are interfering. To do so, we note that, if subcarrier $k$ is time-shared by links $\ell_{1}, \ldots, \ell_{m}$, then the transmissions on links $\ell_{2}, \ldots, \ell_{m}$ interfere with the transmission on link $\ell_{1}$. Hence, the SNIR expression for the transmission on link $\ell_{1}$ is $\frac{q_{\ell_{1} k} g \ell_{\ell_{1} k}}{1+\sum_{i=2}^{m} q_{\ell_{i} k} g_{\ell_{i}^{\prime} k}}$, where $\ell_{i}^{\prime}$ denotes the index of the link connecting the node at which link $\ell_{i}$ originates to the node at which link $\ell_{1}$ ends. Since links $\ell_{1}, \ldots, \ell_{m}$ are simultaneously active on subcarrier $k$ for a fraction of $\gamma_{\ell_{1} \ldots \ell_{m}}^{(k)}$, the expression for the data rate that can be communicated over link $\ell_{1}$ is $\gamma_{\ell_{1} \ldots \ell_{m}}^{(k)} \log _{2}\left(1+\frac{q_{\ell_{1} k} g_{\ell_{1} k}}{1+\sum_{i=2}^{m} q_{\ell_{i} k} g_{\ell_{i}^{\prime} k}}\right)$. Summing over all possible combinations of the interfering links, the capacity constraint on the aggregate flow of link $\ell_{1}$ on subcarrier $k$ can be expressed as

$$
\sum_{d \in \mathcal{D}} x_{\ell_{1} k}^{(d)} \leq \gamma_{\ell_{1}}^{(k)} \log _{2}\left(1+q_{\ell_{1} k} g_{\ell_{1} k}\right)+
$$




$$
\sum_{m=2}^{I} \sum_{\ell_{2} \ldots \ell_{m} \in \mathcal{L}} \gamma_{\ell_{1} \ldots \ell_{m}}^{(k)} \log _{2}\left(1+\frac{q_{\ell_{1} k} g_{\ell_{1} k}}{1+\sum_{i=2}^{m} q_{\ell_{i} k} g_{\ell_{i}^{\prime} k}}\right) .
$$

\section{B. Problem Formulation}

To ensure the feasibility of the rates generated by our design, the constraints in (2)-(12) must be satisfied. Combining these constraints yields the following optimization problem:

$$
\max _{\left\{s_{n}^{(d)}\right\},\left\{x_{\ell k}^{(d)}\right\},\left\{q_{\ell k}\right\}, \Gamma} \sum_{d \in \mathcal{D}} \sum_{n \in \mathcal{N} \backslash\{d\}} w_{n}^{(d)} s_{n}^{(d)},
$$

subject to

Routing constraints in (2)-(4),

Scheduling constraints in (5)-(8),

Power allocation constraints in (10) and (11),

Capacity constraints in (12).

The optimization problem in (13) is nonconvex because of the power allocation constraint in (11) and the capacity constraints in (12). Examining (13) reveals that this problem shares some features with the GP standard form, cf. Appendix A-1 To exploit this observation, in the next section we will perform a change of variables that will enable us to express the objective and all, but one set, of the constraints in a GP-compatible form. The residual constraints that do not comply with the GP standard form are approximated using the monomial approximation technique in Appendix A-2. Under relatively mild conditions [8], iterative application of this technique is known to yield a solution of the KKT system corresponding to [13), see e.g., [5], [19].

\section{Generalized GP-Based Algorithm}

To cast (13) in a form that is amenable to monomial approximation, we define two sets of variables, $\left\{t_{n}^{(d)}\right\}$ and $\left\{r_{\ell k}^{(d)}\right\}$, which are related to $\left\{s_{n}^{(d)}\right\}$ and $\left\{x_{\ell k}^{(d)}\right\}$ by the following maps:

$$
\begin{aligned}
s_{n}^{(d)}=\log _{2} t_{n}^{(d)}, \quad x_{\ell k}^{(d)}=W \log _{2} r_{\ell k}^{(d)}, \\
n \in \mathcal{N} \backslash\{d\}, d \in \mathcal{D}, \ell \in \mathcal{L}, k \in \mathcal{K} .
\end{aligned}
$$

These maps are bijective, which renders recovering $\left\{s_{n}^{(d)}, x_{\ell k}^{(d)}\right\}$ straightforward. The objective and the routing constraints in (13) can be cast in a GP-compatible form. In particular, the objective can be expressed as $\prod_{d \in \mathcal{D}} \prod_{n \in \mathcal{N} \backslash\{d\}}\left(t_{n}^{(d)}\right)^{w_{n}^{(d)}}$ and the routing constraints can be expressed as

$$
\begin{array}{ll}
\prod_{\ell \in \mathcal{L}} \prod_{k \in \mathcal{K}}\left(r_{\ell k}^{(d)}\right)^{W a_{n \ell}}=t_{n}^{(d)}, & n \in \mathcal{N} \backslash\{d\}, d \in \mathcal{D}, \\
r_{\ell k}^{(d)} \geq 1, & \ell \in \mathcal{L}, k \in \mathcal{K}, d \in \mathcal{D}, \\
t_{n}^{(d)} \geq 1, & n \in \mathcal{N} \backslash\{d\}, d \in \mathcal{D} .
\end{array}
$$

The non-negativity constraints in (5) and (10) are inherently satisfied in the GP framework. The constraints in (6) and (11) are already in a GP-compatible form. We now consider the constraints in (7) and (8). The right hand side (RHS) of these constraints are zero, which makes them incompatible with the GP framework in Appendix A-1 This problem can be alleviated by constraining their LHS to be less than an arbitrary small number $\epsilon>0$, i.e.,

$$
\begin{aligned}
& a_{n \ell_{1}}^{+} a_{n \ell_{2}}^{+}\left(\gamma_{\ell_{1} \ell_{2}}^{(k)}+\sum_{m=3}^{I} \sum_{\substack{\ell_{3} \cdots \ell_{m} \in \mathcal{L} \\
\ell_{1} \in \mathcal{L}, \ell_{2} \in \mathcal{L} \backslash\left\{\ell_{1}\right\}, k \in \mathcal{K},}} \gamma_{\ell_{1} \ldots \ell_{m}}^{(k)}\right) \leq \epsilon, \\
& a_{n \ell_{1}}^{+} a_{n \ell_{2}}^{-}\left(\gamma_{\ell_{1} \ell_{2}}^{(k)}+\sum_{m=3}^{I} \sum_{\substack{\ell_{3} \cdots \ell_{m} \in \mathcal{L} \\
\ell_{1} \in \mathcal{L}, \ell_{2} \in \mathcal{L} \backslash\left\{\ell_{1}\right\}, k \in \mathcal{K} .}} \gamma_{\ell_{1} \ldots \ell_{m}}^{(k)}\right) \leq \epsilon,
\end{aligned}
$$

The remaining constraints that are not GP-compatible are those in (12). Invoking the change of variables in (14), for $\ell_{1} \in \mathcal{L}$ and $k \in \mathcal{K}$, those constraints can be expressed as

$$
\begin{aligned}
\prod_{d \in \mathcal{D}} r_{\ell_{1} k}^{(d)} \leq & \left(1+q_{\ell_{1} k} g_{\ell_{1} k}\right)^{\gamma_{\ell_{1}}^{(k)} \times} \\
& \prod_{m=2}^{I} \prod_{\ell_{2} \cdots \ell_{m} \in \mathcal{L}}\left(1+\frac{q_{\ell_{1} k} g_{\ell_{1} k}}{1+\sum_{i=2}^{m} q_{\ell_{i} k} g_{\ell_{i}^{\prime} k}}\right)^{\gamma_{\ell_{1} \cdots \ell_{m}}^{(k)}} .
\end{aligned}
$$

The RHS of (20) is amenable to the monomial approximation technique described in Appendix A-2 [7]. One approach to use this technique is to approximate all the terms in the RHS of 201 by one monomial. This approach is overly complicated, and an alternative is to approximate each term by a monomial. The product of these monomials constitutes a monomial approximation of the RHS of (20). Hence, the constraint in 20) can be approximated with

$$
\begin{aligned}
& \prod_{d \in \mathcal{D}} r_{\ell_{1} k}^{(d)} \leq \mathcal{M}\left(\left(1+q_{\ell_{1} k} g_{\ell_{1} k}\right)^{\gamma_{\ell_{1}}^{(k)}}\right) \times \\
& \prod_{m=2}^{I} \prod_{\ell_{2} \ldots \ell_{m} \in \mathcal{L}} \mathcal{M}\left(\left(1+\frac{q_{\ell_{1} k} g_{\ell_{1} k}}{1+\sum_{i=2}^{m} q_{\ell_{i} k} g_{\ell_{i}^{\prime} k}}\right)^{\gamma_{\ell_{1} \ldots \ell_{m}}^{(k)}}\right),
\end{aligned}
$$

where the functional $\mathcal{M}(\cdot)$ is described in Appendix A-2 Note that, $\left\{\gamma_{\ell_{1} \ldots \ell_{m}}^{(k)}\right\}$ are variables and hence inseparable from the argument of $\mathcal{M}(\cdot)$.

Now, the problem in (13) can be approximated by the following GP:

$$
\max _{\left\{t_{n}^{(d)}\right\},\left\{r_{\ell k}^{(d)}\right\},\left\{q_{\ell k}\right\}, \Gamma} \prod_{d \in \mathcal{D}} \prod_{n \in \mathcal{N} \backslash\{d\}}\left(t_{n}^{(d)}\right)^{w_{n}^{(d)}},
$$

subject to

Routing constraints in (15)-(17),

Scheduling constraints in (6), (18) and (19),

Power allocation constraints in (11),

Approximate capacity constraints in 21).

Note that the relaxations in (18) and (19) may result in infeasible subcarrier time-shares that do not satisfy the constraints in (7) and (8). To construct feasible schedules, the elements of $\Gamma$ that are less than or equal to $\epsilon$ are set to zero. Using a standard exponential transformation, the GP in 22 can be readily transformed into a convex optimization problem which can be solved in polynomial time using interior-point 
TABLE II: Successive GP-based Algorithm for Solving 22

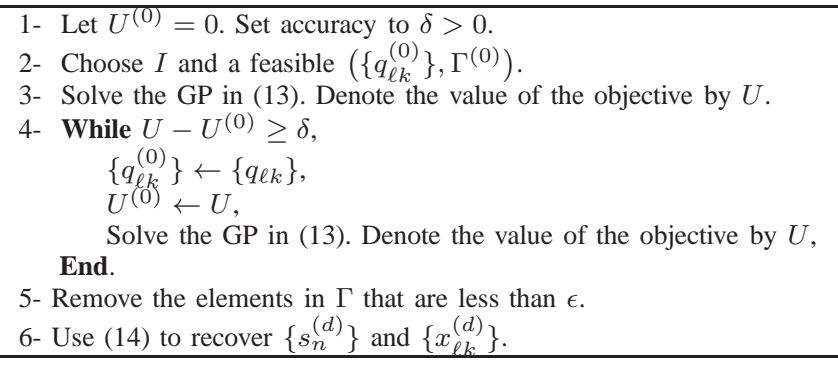

methods (IPMs) [7]. This implies that (22) enables us to efficiently solve (13) approximately in the neighbourhood of any initial set $\left(\left\{q_{\ell k}^{(0)}\right\}, \Gamma^{(0)}\right)$.

Finding the global solution for the nonconvex problem in 13 is difficult, whereas solving the approximated problem in (22) is straightforward. To exploit this fact, we incorporate the formulation in (22) in an iterative algorithm, whereby the output of solving (22) for an initial point $\left(\left\{q_{\ell k}^{(0)}\right\}, \Gamma^{(0)}\right)$ is used as a starting point for the subsequent iteration. This technique is usually referred to as the single condensation method, e.g., [19], [20], and under relatively mild conditions, its convergence to a solution of the KKT system corresponding to (13) is guaranteed [8]. Since the original design problem is not convex, this system has multiple local solutions and the one to which the single condensation method converges depends on the initial point; some of the local solutions may be global ones. A summary of this algorithm is described in Table [1]

In the next section, we will discuss a special case of this algorithm when time-sharing of subcarriers is not allowed. Before we do that, we now provide a brief discussion on the implementation of this algorithm. To begin with, we note that the algorithm in Table $\Pi$ is centralized, in the sense that the design is performed by a central entity that is aware of the network parameters. The signalling exchange between the nodes and the central entity, required to establish communication in the considered framework, are described as follows. At the beginning of each signalling interval, the central entity prompts the nodes in the network to sequentially broadcast pilot signals of prescribed power levels. Subsequently, each node computes the subcarrier channel gains from all other nodes in the network. There is total of $L K$ such gains, where $L$ is the number of links and $K$ is the number of subcarriers. Each node sends these gains along with its destination nodes, if any, and its priority weights to the central entity. The central entity performs the joint optimization of the power allocations, scheduling parameters and data routes as described in Table It then forwards these decisions to all the nodes, possibly over a dedicated control channel. In particular, the information forwarded by the central entity include 1) the subcarrier index and the time allocated to each transmission. This information is provided by the set $\Gamma$. The cardinality of this set depends on the number of simultaneous transmissions allowed in each subcarrier, $I$. For instance, for $I=2,|\Gamma|=L K(L+1) / 2$; 2) The power allocated to each transmission. This information is provided by the set $\left\{q_{\ell k}\right\}$ and the cardinality of this set is $L K$; and 3) The data rates at each transmitting and receiving node in the route of the stream intended for each destination. This information is provided in the set $\left\{x_{\ell k}^{(d)}\right\}$ and the cardinality of this set is $L K D$, where $D$ is the number of intended destinations.

\section{Special Case: Routing and Resource ALLOCATION WITHOUT TIME-SHARING}

In this section, we consider a design problem similar to the one described in Section [V] but for the case when timesharing of subcarriers is not allowed. This corresponds to the a special case in which the entries of $\Gamma$ in Section IV-A2 are restricted to be binary. This restriction results in a mixed integer program which is generally difficult to solve. To overcome this difficulty, we capture the effect of the scheduling variables in the power allocation constraints. We will show that this approach will enable us to develop a design algorithm with polynomial-complexity.

\section{A. System Constraints}

1) Routing Constraints: These constraints are identical to those described in (2)-(4).

2) Power Allocation Constraints: In characterizing these constraints, we will use the method described in Section V-A2 to denote the power allocated for transmission on subcarrier $k$ of link $\ell$ by the variables $\left\{q_{\ell k}\right\}$. These variables must satisfy the non-negativity constraints in (10) and the power budget constraint. These constraints, using (9), can be cast as

$$
\sum_{k \in \mathcal{K}} \sum_{\ell \in \mathcal{L}} a_{n \ell}^{+} q_{\ell k} \leq P_{n}, \quad n \in \mathcal{N} .
$$

Similar to the case considered in Section IV] the nodes cannot simultaneously broadcast to multiple destinations on the same subcarrier. However, this requirement in the current case can be implicitly captured by the allocation of the link powers. In particular, for any subcarrier $k \in \mathcal{K}$, any node $n \in \mathcal{N}$ and any two links $\ell_{1}, \ell_{2} \in \mathcal{L}_{+}(n)$, at least $q_{\ell_{1} k}=0$ or $q_{\ell_{2} k}=0$, i.e.,

$$
a_{n \ell_{1}}^{+} a_{n \ell_{2}}^{+} q_{\ell_{1} k} q_{\ell_{2} k}=0, \quad \ell_{1}, \ell_{2} \in \mathcal{L}, k \in \mathcal{K}, n \in \mathcal{N} .
$$

This constraint is significantly less involved than the one in (7).

Similarly, the half-duplex requirement can be captured by ensuring that, for each node $n \in \mathcal{N}$, if the power on subcarrier $k$ of $\mathcal{L}_{+}(n)$ is strictly positive, then the power allocated to this subcarrier on all the links in $\mathcal{L}_{-}(n)$ is zero, and vice versa. Hence, the half-duplex requirement can enforced by the following constraints:

$$
a_{n \ell_{1}}^{-} a_{n \ell_{2}}^{+} q_{\ell_{1} k} q_{\ell_{2} k}=0, \quad \ell_{1}, \ell_{2} \in \mathcal{L}, k \in \mathcal{K}, n \in \mathcal{N} .
$$

Note that these constraints are simpler than their counterparts in (8). Also, note that (24) and (25) are trivially satisfied if either link $\ell_{1}$ or $\ell_{2}$ are not connected to node $n$.

3) Capacity Constraints: In this case, the constraints in 12 can be readily seen to reduce to

$$
\sum_{d} \frac{x_{\ell_{1} k}^{(d)}}{W} \leq \log _{2}\left(1+\frac{q_{\ell_{1} k} g_{\ell_{1} k}}{1+\sum_{\ell_{2} \in \mathcal{L} \backslash\left\{\ell_{1}\right\}} q_{\ell_{2} k} g_{\ell_{2}^{\prime} k}}\right) .
$$




\section{B. Problem Formulation}

Using the characterization described in Section $\mathrm{V}-\mathrm{A}$, the design problem can be cast as:

$$
\max _{\left\{s_{n}^{(d)}\right\},\left\{x_{\ell k}^{(d)}\right\},\left\{q_{\ell k}\right\}} \sum_{d \in \mathcal{D}} \sum_{n \in \mathcal{N} \backslash\{d\}} w_{n}^{(d)} s_{n}^{(d)},
$$

subject to

Routing constraints in (2)-(4),

Power allocation constraints in (10), 23)- 25),

Capacity constraints in 26.

The optimization problem in 27) is nonconvex because the RHS of 26 is the logarithm of a rational function, and therefore not concave. The equality constraints in (24) and 25 are not affine and hence, nonconvex. In the next section, we will develop a GP-based algorithm, analogous to the one described in Section IV-C to obtain a locally optimal solution.

\section{Proposed GP-based Algorithm}

The optimization problem in (27), although nonconvex, is amenable to the GP-based monomial approximation in Appendix A-2 To use this approximation, we use (14) to transform $\left\{s_{n}^{(d)}\right\}$ and $\left\{x_{\ell k}^{(d)}\right\}$ to $\left\{t_{n}^{(d)}\right\}$ and $\left\{r_{\ell k}^{(d)}\right\}$, respectively. Using these new variables, the routing constraints are readily expressed in a GP-compatible form as described in (15)-17).

Substituting from (14) into (26) yields the following set of equivalent constraints:

$$
\begin{aligned}
& \left(1+\sum_{\ell_{2} \in \mathcal{L} \backslash\left\{\ell_{1}\right\}} q_{\ell_{2} k} g_{\ell_{2}^{\prime} k}\right) \prod_{d \in \mathcal{D}} r_{\ell_{1} k}^{(d)} \leq 1+\sum_{\ell_{2} \in \mathcal{L}} q_{\ell_{2} k} g_{\ell_{2}^{\prime} k}, \\
& k \in \mathcal{K}, \ell_{1} \in \mathcal{L},
\end{aligned}
$$

which are, unfortunately not GP-compatible. Using the monomial approximation technique in Appendix A-2 yields the following approximation of (28) in the neighbourhood of $\left\{q_{\ell k}^{(0)}\right\}$ :

$$
\begin{gathered}
\left(1+\sum_{\ell_{2} \in \mathcal{L} \backslash\left\{\ell_{1}\right\}} q_{\ell_{2} k} g_{\ell_{2}^{\prime} k}\right) \prod_{d \in \mathcal{D}} r_{\ell_{1} k}^{(d)} \leq c_{\ell_{1} k} \prod_{\ell_{2} \in \mathcal{L}}\left(q_{\ell_{2} k} / q_{\ell_{2} k}^{(0)}\right)^{\theta_{\ell_{2} k}}, \\
k \in \mathcal{K}, \ell_{1} \in \mathcal{L}, \quad(29)
\end{gathered}
$$

where $\left\{q_{\ell k}^{(0)}\right\}$ is the initial power allocation, $c_{\ell_{1} k}=1+$ $\sum_{\ell_{2} \in \mathcal{L}} q_{\ell_{2} k}^{(0)} g_{\ell_{2}^{\prime} k}$, and $\theta_{\ell_{2} k}=q_{\ell_{2} k}^{(0)} g_{\ell_{2}^{\prime} k} / c_{\ell_{1} k}$.

Analogous to the case considered in Section IV-C 24 and 25) are replaced with the GP-compatible inequality constraints. The joint design of data routes and power allocations in (27) can be approximated with the following GP:

$$
\max _{\left\{t_{n}^{(d)}\right\},\left\{r_{\ell k}^{(d)}\right\},\left\{q_{\ell k}\right\}} \prod_{d \in \mathcal{D}} \prod_{n \in \mathcal{N} \backslash\{d\}}\left(t_{n}^{(d)}\right)^{w_{n}^{(d)}},
$$

subject to

Routing constraints in (15)-17,

Power allocation constraints in 23-25 (relaxed versions),

Approximate capacity constraints in 29.

A locally optimal solution of (27) can be obtained by solving (30) iteratively using the single condensation method described in Section IV-C

\section{COMPlEXity AnAlysis}

In this section we examine the computational complexity required for solving the problems described in Sections IV-C and $\mathrm{V}-\mathrm{C}$ for the cases with and without time-sharing, respectively. The algorithms in these sections iteratively solve the families of the optimization problems in (22) and (30). Being in a GP-compatible form, these problems can be readily converted into convex forms and can be efficiently solved using IPM-based solvers.

In IPM, the objective and inequality constraints are used to construct a log-barrier function which is minimized along a central path using Newton's method. The complexity of each Newton step grows with the cube of the number of inequality constraints and the number of Newton steps can be bounded if the log-barrier function is self-concordant [21], cf. Appendix B. In that case, the number of Newton steps can be shown to grow with the square root of the number of inequality constraints [21].

Unfortunately, the log-barrier functions related to the problems in (22) and (30) are not self-concordant. To circumvent this difficulty, we introduce a set of auxiliary variables and constraints which, although redundant, enables us to construct self-concordant log-barrier functions. Using these functions and the results in [21], we arrive at the following proposition:

Proposition 1. The complexity of solving with IPM-based solvers is of order

$$
\mathcal{O}\left(\left(2 L K N+N+D(N-1)+2 K \sum_{i=1}^{I}\left(\begin{array}{l}
L \\
i
\end{array}\right)\right)^{3.5}\right),
$$

and the complexity of solving with IPM-based solvers is of order

$$
\mathcal{O}\left((L K(3 L+2)+N+D(N-1))^{3.5}\right) .
$$

Proof: See Appendix B

The first statement of Proposition 1 pertains to the general case with time-sharing and frequency-reuse. This statement shows that the complexity of solving the problem in 22 is polynomial in $L$ for small values of $I$. The complexity of solving (22) can be further reduced by combining the broadcasting constraint and the half-duplex constraint in (7) and (8), respectively. In particular, examining these constraints reveals that they are related to the network topology and do not depend on the channel conditions. Hence, these two constraints can be enforced by pruning the set $\Gamma$ prior to solving 13 or its approximated version in 22. The pruning rule is as follows: For each $\ell$ and $\ell^{\prime} \in \mathcal{L}$, if either $a_{n \ell_{1}}^{+} a_{n \ell_{2}}^{+}=0$ or $a_{n \ell_{1}}^{+} a_{n \ell_{2}}^{-}=0$, the corresponding time-shares in (7) and (8) are removed from the set $\Gamma$. Unfortunately, we have not been able to obtain a closed form of the cardinality of the resulting $\Gamma$. However, the reduction in complexity, at least for small networks, appears to be significant. For instance, for fully connected networks with $N=4$ nodes and $L=N(N-1)=12$ links, $|\Gamma|$ is reduced from 4095 to 40.

The second statement of Proposition 1 pertains to the special case in which time-sharing is not allowed. This statement shows that the complexity of solving (30) is polynomial in the 
TABLE III: Normalized Channel Gains, $\left\{g_{\ell k}\right\}$, in Example 1 [dB].

\begin{tabular}{l|cccccc} 
& link 1 & link 2 & link 3 & link 4 & link 5 & link 6 \\
\hline subcarrier 1 & -6.1 & -11.1 & 1.86 & -12.3 & -13.5 & 42.4 \\
subcarrier 2 & -5.7 & 1.57 & 5.64 & -5.98 & -19.2 & 40.7 \\
\hline & link 7 & link 8 & link 9 & link 10 & link 11 & link 12 \\
\hline subcarrier 1 & 15.82 & -2.43 & -5.82 & -6.9 & 35.0 & 3.304 \\
subcarrier 2 & -3.38 & -5.61 & 0.871 & -0.6 & 41.2 & -14.5
\end{tabular}

number of nodes, $N$, and the number of subcarriers, $K$. In particular, it grows as $L^{7} K^{3.5}$. Another case in which the design complexity is polynomial is the one in which the subcarriers are time-shared but not frequency-reused [5]. In that case the design complexity is $\mathcal{O}\left((L K(4+D)+N+K+D(N-1))^{3.5}\right)$. Hence the special cases with either no frequency-reuse or no time-sharing have polynomial complexity.

\section{Simulation Results}

In this section we provide numerical results to evaluate the performance of joint routing and resource allocation algorithms for the cases with and without time-sharing.

The locations of the nodes are randomly generated and evenly distributed over a $300 \times 300 \mathrm{~m}^{2}$ square. The nodes are assumed to have identical power budgets, i.e, $P_{n}=P, \forall n \in$ $\mathcal{N}$, and the available frequency-selective channel is partitioned into a set of frequency-flat Rayleigh fading channels with the values of pathloss (PL) and shadowing components obtained from the non line-of-sight communication of indoor hotspot (InH) scenario in the IMT-Advanced document [22]. According to [22], the PL component on link $\ell \in \mathcal{L}$ is given by

$$
P L=43.3 \log _{10}\left(d_{\ell}\right)+11.5+20 \log _{10}\left(f_{c}\right),
$$

where $d_{\ell}$ is the length of link $\ell$ in meters and $f_{c}$ is the carrier frequency in Gigahertz which, in our simulations, is set to $f_{c}=3.4 \mathrm{GHz}$. The shadowing component is assumed to be log-normal distributed with a mean of $0 \mathrm{~dB}$ and a standard deviation of $4 \mathrm{~dB}$. The Rayleigh fading component is generated by the envelope of a zero-mean unit-variance complex Gaussian-distributed random variable. The available bandwidth around each subcarrier is set to $W=200 \mathrm{KHz}$ and the noise power density at receivers is set to $N_{0}=$ $-174 \mathrm{dBm} / \mathrm{Hz}$.

The results reported herein are obtained using the CVX package [23] with an underlying MOSEK solver [24]. The value of $\epsilon$ in (7) and (8) is set to $10^{-4}$.

Example 1: (Joint Routing and Resource Allocation with Time-sharing) Consider an exemplary network with $N=4$ nodes. In this network, nodes 3 and 4 wish to communicate with nodes 2 and 1, respectively, over $K=2$ subcarriers. In particular, for destination node $d=1$, the source is node $n=4$ and nodes $\{2,3\}$ are potential relays, and, for destination node $d=2$, the source is node $n=3$ and nodes $\{1,4\}$ are potential relays. The considered network has $L=12$ directional links and therefore the channel matrix has $12 \times 2$ elements. The channels are assumed to be static and their normalized gain in $\mathrm{dB}$, i.e., $10 \log _{10} g_{\ell k}$, is given in Table III In this example, the power budget of each node is set to $P=20 \mathrm{dBm}$, the number of simultaneous transmissions is set to $I=3$ and the two
TABLE IV: Power Allocations (mW) in Example 1.

\begin{tabular}{|l|l|l|l|}
\hline$n=1$ & $n=2$ & $n=3$ & $n=4$ \\
\hline$q_{3,2}=23$ & $q_{4,2}=56.5$ & $q_{7,1}=6.5$ & $q_{10,2}=24.5$ \\
& & $q_{9,2}=13.5$ & $q_{11,1}=0.3$ \\
& & $q_{12,1}=16.5$ \\
\hline
\end{tabular}

rates, $s_{3}^{(2)}$ and $s_{4}^{(1)}$, are assigned equal weights, i.e., $w_{3}^{(2)}=$ $w_{4}^{(1)}=1$. Since in this example time-sharing is allowed, the algorithm in Section IV-C is used to generate the data routes, time-sharing schedules and power allocations.

The sum-rate yielded by the algorithm in Section IV-C is $7.4 \mathrm{~b} / \mathrm{s} / \mathrm{Hz}$. The data routes generated by this algorithm are illustrated in Figure 3. For ease of exposition, the network in this example is split into the two sub-networks: the one in Figure 3(a) depicts the routes of the data intended for destination $d=1$, and the one in Figure 3(b) depicts the routes of the data intended for destination $d=2$. The complete network is the superposition of the two sub-networks. For instance, the data transmitted over link 7 , connecting node 3 to node 1 , is $4.8 \mathrm{~b} / \mathrm{s} / \mathrm{Hz}$, of which $2 \mathrm{~b} / \mathrm{s} / \mathrm{Hz}$ is intended for destination $d=1$ and $2.8 \mathrm{~b} / \mathrm{s} / \mathrm{Hz}$ is intended for destination $d=2$.

The time-sharing schedules of the subcarriers generated by the algorithm in Section IV-C are provided in Figure 4 It can be seen from this figure that subcarrier $k=1$ is both reused and time-shared, whereas subcarrier $k=2$ is only time-shared. Figures 3 and 4 imply that link 7 , connecting node 3 to node 1 , and link 11 , connecting node 4 to node 2 , carry the data intended for both destinations on the same subcarrier, $k=1$, during the same time interval. The fact that our designs enforce half-duplex requirement can be inferred from these figures. For instance, Figure 3(a) shows that node 3 uses the same subcarrier, $k=1$, for its transmission and reception on links 7 and 12, respectively, but Figure 4 shows that transmission and reception occur during different time intervals.

The power allocations yielded by the algorithm in Section IV-C are shown in Table IV This table shows that, because of frequency-reuse, the nodes do not necessarily use their total power budgets. This is due to the fact that, in this scenario, when a node increases its transmission power, it inflicts high interference on other transmissions. This is in contrast with the situation considered in [5], wherein frequency-reuse is not allowed and increasing the transmitted power of a node does not affect the transmissions of the other nodes in the network.

Example 2: (Joint Routing and Resource Allocation without Time-sharing) Consider an exemplary network with $N=6$ nodes. In this network, as before, nodes 3 and 4 wish to communicate with nodes 2 and 1 , respectively, over $K=4$ subcarriers. In particular, for destination node $d=1$, the source is node $n=4$ and the other nodes, i.e., $\{2,3,4,5\}$ are potential relays, and, for destination node $d=2$, the source is node $n=3$ and nodes $\{1,4,5,6\}$ are potential relays.

The considered network has $L=30$ links and therefore the channel matrix has $30 \times 4$ elements. For space considerations, this matrix is not provided, but since the channel gain on each subcarrier is dominated by the PL component, we provide the coordinates of the nodes in the $300 \times 300 \mathrm{~m}^{2}$ square; calculating the PL components from these coordinates is 


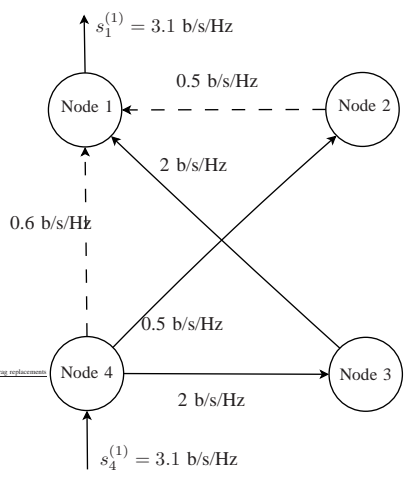

(a)

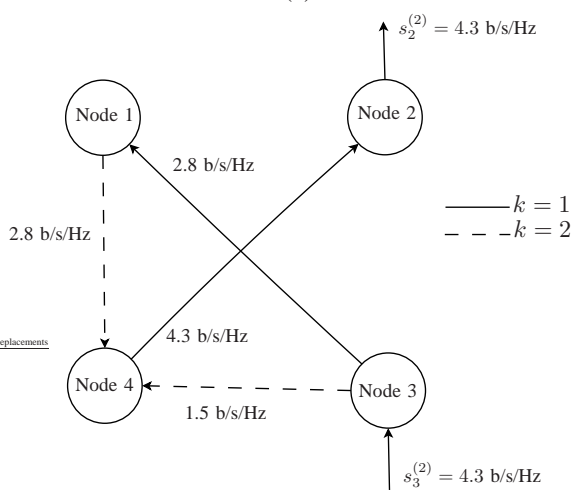

(b)

Fig. 3: Data routes for (a) $d=1$, (b) $d=2$ in Example 1 .

\begin{tabular}{|c|c|c|c|c|c|}
\hline \multicolumn{3}{|c|}{$k=1$} & \multicolumn{3}{|c|}{$k=2$} \\
\hline \multirow{3}{*}{$\begin{array}{l}\text { Link } 7 \\
\text { Link } 11 \\
\text { Link } 12\end{array}$} & $60 \%$ & & \multirow{4}{*}{$\begin{array}{l}\text { Link } 3 \\
\text { Link } 4 \\
\text { Link } 9 \\
\text { Link } 10\end{array}$} & $45 \%$ & \\
\hline & $60 \%$ & & & & \\
\hline & & $40 \%$ & & & $30 \%$ \\
\hline & & & & & \\
\hline
\end{tabular}

Fig. 4: Time-sharing schedules of the subcarriers in Example 1.

straightforward, cf. 31]. The coordinates of the nodes are $\{(283,202),(191,208),(287,20),(72,76),(201,67),(86,200)\}$

Setting the node power budgets to $P=20 \mathrm{dBm}$ and assuming that both rates have equal weights, $w_{3}^{(2)}=w_{4}^{(1)}=1$, the joint design algorithm in Section $\mathrm{V}-\mathrm{C}$ yields a sum-rate of $9.1 \mathrm{~b} / \mathrm{s} / \mathrm{Hz}$. The data routes and power allocations obtained by this algorithm are shown in Figure 5 and Table $\mathrm{V}$, respectively. For instance, in Figure 5, subcarrier $k=1$ is shown to be used twice and due to the half-duplex constraint, transmission and reception take place over distinct subcarriers at each node. We will later show the advantage of the proposed algorithm over the algorithms in which frequency-reuse is not considered.

Example 3: (Average Weighted-Sum Rate Comparison) In this example, we use Monte Carlo simulations to evaluate the average performance of the joint designs with and without time-sharing when the channels are time-varying rather than

TABLE V: Power Allocations (mW) in Example 2.

\begin{tabular}{|l|l|l|l|}
\hline$n=1$ & $n=3$ & $n=4$ & $n=5$ \\
\hline$q_{1,1}=12$ & $q_{11,2}=45$ & $q_{16,4}=100$ & $q_{22,3}=25$ \\
& $q_{14,1}=55$ & & \\
\hline
\end{tabular}

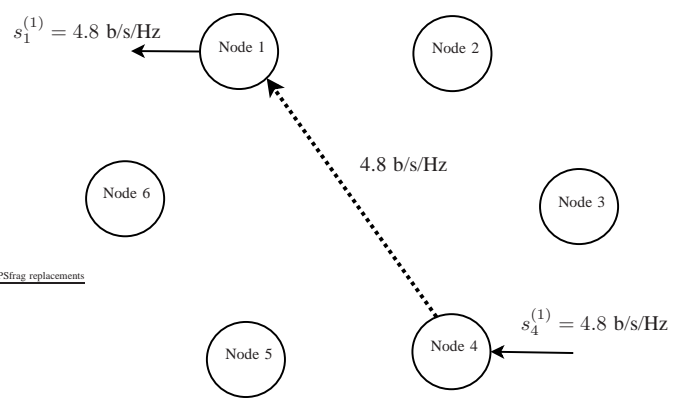

(a)

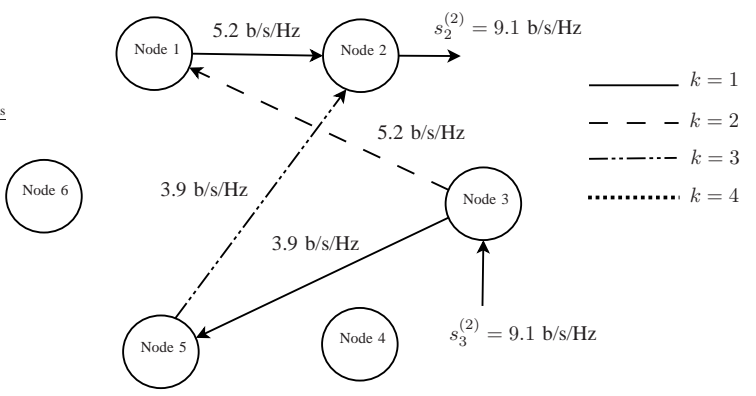

(b)

Fig. 5: Data routes for (a) $d=1$, (b) $d=2$ in Example 2.

static as in Examples 1 and 2. We consider a network with $N=4$ nodes in which nodes 3 and 4 wish to communicate with nodes 2 and 1 , respectively, over $K=4$ subcarriers. The number of simultaneous transmissions is set to $I=3$ and the simulation results are averaged over 10 independent network realizations.

The average weighted-sum rates yielded by the algorithms in Sections $\mathrm{V}-\mathrm{C}$ and $\mathrm{IV}-\mathrm{C}$ for the values of $P$ ranging from 0 to $30 \mathrm{dBm}$ are depicted in Figures 6(a) and 6(b) for the cases of $w_{3}^{(2)}=5 w_{4}^{(1)}$ and $w_{3}^{(2)}=w_{4}^{(1)}$, respectively. These figures also provide a comparison with the weighted-sum rates yielded by the designs in which frequency-reuse is not considered [5].

As can be seen from Figure 6, the weighted-sum rate yielded by the joint design with both time-sharing and frequencyreuse outperforms the designs in which either time-sharing or frequency-reuse is exclusively considered, but at the expense of increased complexity. For instance, Figure 6(b) suggests that, at the sum-rate of $12 \mathrm{~b} / \mathrm{s} / \mathrm{Hz}$, the proposed design with both time-sharing and frequency-reuse yields a power advantage of $4 \mathrm{dBm}$ over the designs in which either time-sharing or frequency-reuse is exclusively considered and a power advantage of $8 \mathrm{dBm}$ over the design in which neither of these techniques is considered. This figure also suggests that, for values of $P$ less than $15 \mathrm{dBm}$, the design with frequency-reuse but without time-sharing yields better performance than the design with time-sharing but without frequency-reuse in [5]. However, for values of $P$ higher than $15 \mathrm{dBm}$, the design with time-sharing but without frequency-reuse performs better than the one with frequency-reuse but without time-sharing. This phenomenon can be attributed to the effect of interference. At low powers, the effect of interference is small and frequencyreuse performs generally better than time-sharing. In contrast, at high powers, the effect of interference is more severe and time-sharing performs generally better than frequency- 


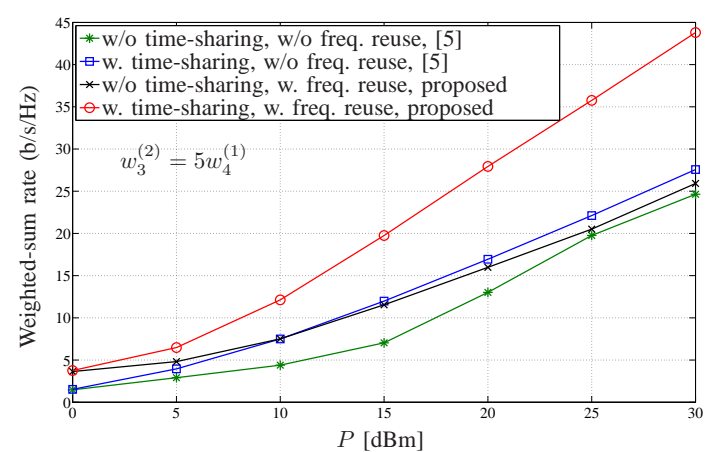

(a)

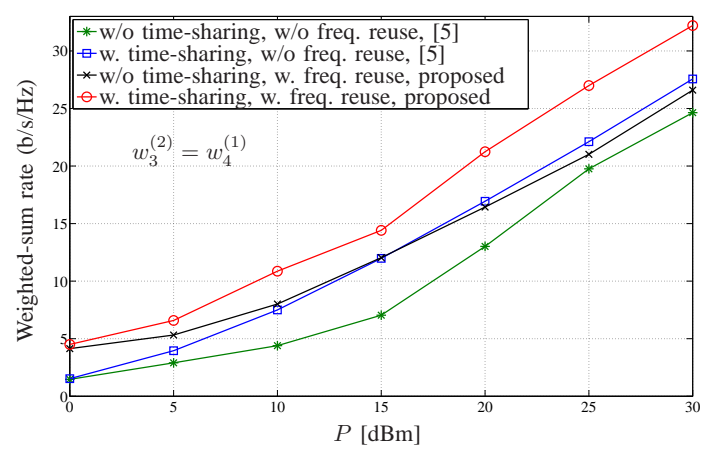

(b)

Fig. 6: Average weighted-sum rate comparison for (a) $w_{3}^{(2)}=5 w_{4}^{(1)}$, and (b) $w_{3}^{(2)}=w_{4}^{(1)}$.

reuse. As expected, the design with neither time-sharing nor frequency-reuse has inferior performance.

Example 4: (Joint Routing and Resource Allocation: Generalized Algorithm) In this example, we evaluate the performance of the algorithm developed in Section IV-C. We consider a snapshot of a network with $N=5$ nodes and $L=20$ links (links with a distance more than $150 \mathrm{~m}$ are neglected). In this network nodes 3 and 4 wish to communicate with nodes 2 and 1 , respectively, over $K=4$ subcarriers. The number of simultaneous transmissions is set to $I=20, I=3$ and $I=2$, which results in $\Gamma$ with $2^{20}-1,190$ and 110 variables, respectively.

The sum-rate yielded by the generalized algorithm with different values of $I$ is depicted in Figure 7 For comparison, this figure also shows the rates yielded by the special case in Section $\mathrm{V}$. As can be seen from Figure 7, the algorithm with $I=2$ and 3 yields rates that are slightly less than the rate yielded by the algorithm with $I=L$, however with a significantly less computational complexity. In fact, the complexity of the algorithm with $I \ll L$ is polynomial, whereas that of the algorithm with $I=L$ is exponential in $L$. This feature renders the algorithm with $I \leq 3$ more attractive for designing large networks with potentially rapid channel variations. From Figure 7 it can be seen that the gap between the rates yielded by different values of $I$ decreases as the power budget increases. This is because as power increases, interference becomes more severe, which causes the reuse of a particular subcarrier on multiple links less beneficial. It can be also seen from this figure that, most of the gain of frequency-reuse is mustered by only considering two or three simultaneous transmissions, i.e., $I \leq 3$. This implies that increasing $I$ trades complexity for performance. In particular, as $I$ increases, the performance of the algorithm becomes closer to that of the one with $I=L$, but at the expense of increased complexitv.

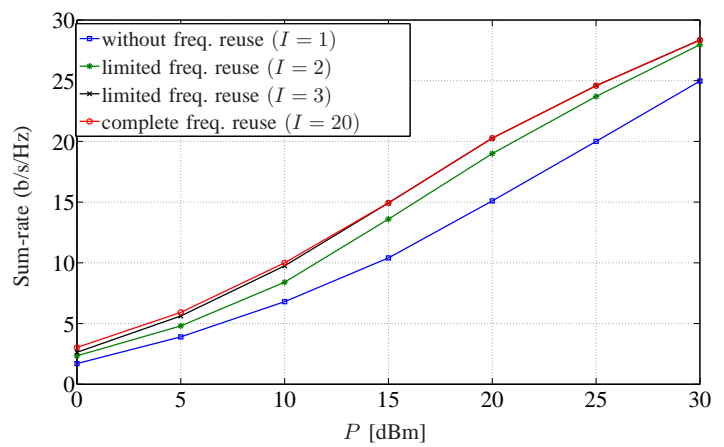

Fig. 7: Sum-rate generated by the generalized algorithm for different values of $I$.

In Figure 8(a) we investigate the convergence behaviour of the generalized algorithm. We consider an instance of a network in which the power budget of all nodes is set to $P=10 \mathrm{dBm}$. It can be seen from this figure that, in addition to being significantly less computationally demanding, the algorithm with a lower value of $I$ exhibits considerably faster convergence than that of the one with a higher value of $I$. This convergence can be further ameliorated by choosing the initial point more carefully, for instance, by choosing this point to be the solution yielded by algorithm in [5] for the case with no frequency-reuse.

To illustrate the effect of random initialization of the generalized algorithm, in Figure 8(b) the value of the objective to which the generalized algorithm with $I=2$ converged is shown for 80 random instances of feasible initial points, $\left(q_{\ell k}^{(0)}, \Gamma^{(0)}\right) \in[0, P]^{L K} \times[0,1]^{|\Gamma|}$. It can be seen from this figure that although the algorithm is relatively sensitive to the choice of the initial point, finding initial points that result in 'good' local maxima is generally easy.

Example 5: (Average Rate-Region Comparison) In this example we provide the rate regions that can be achieved by the algorithms in Sections $\mathrm{V}-\mathrm{C}$ and $\mathrm{IV}-\mathrm{C}$ when $P=10 \mathrm{dBm}$. These regions are obtained by varying the weights $\left(s_{4}^{(1)}, s_{3}^{(2)}\right)$ over the unit simplex, i.e., $\left\{\left(w_{4}^{(1)}, w_{3}^{(2)}\right) \mid w_{4}^{(1)} \geq 0, w_{3}^{(2)} \geq\right.$ $\left.0, w_{4}^{(1)}+w_{3}^{(2)}=1\right\}$, and are depicted in Figure9 A comparison between these rate regions and the ones corresponding to the case when frequency-reuse is not considered [5] is also provided in this figure. As can be seen from Figure 9 , the rate region corresponding to the design with both timesharing and frequency-reuse properly contains the rate regions corresponding to the designs in which either time-sharing or frequency-reuse is exclusively used. It can be also seen that restricting the number of simultaneous transmissions to be less than three suffices to achieve most of the frequency-reuse gain and with less computational complexity.

\section{CONCLUSION}

In this paper we focused on the joint optimization of data routes, subcarrier schedules and power allocation in a 


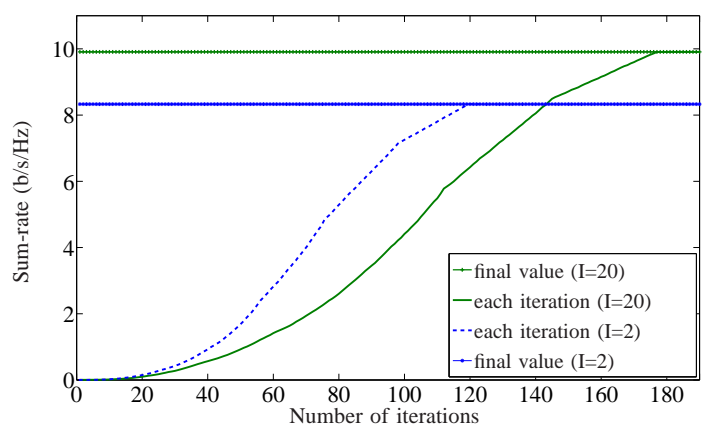

(a)

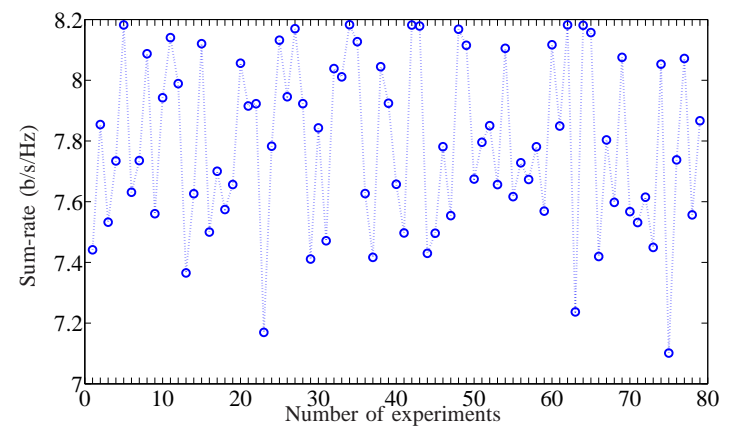

(b)

Fig. 8: (a) Convergence behaviour and (b) performance of the generalized algorithm with different initial points.

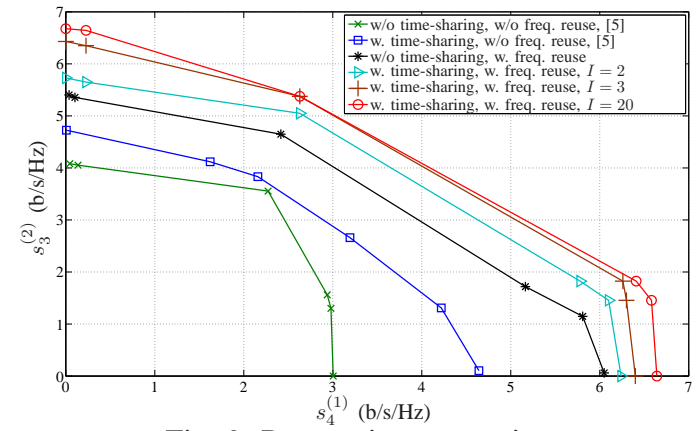

Fig. 9: Rate-region comparison.

half-duplex multicarrier network when each subcarrier can be reused by multiple links. The goal is to maximize a weighted-sum of the rates communicated over the network. The considered network is generic in the sense that it subsumes many structures including cellular and device-to-device communications as special cases. We considered two instances of this problem: 1) when each subcarrier can be time-shared by multiple links; and 2) when time-sharing is not allowed and a subcarrier, once assigned to a set of links, will be used by those links throughout the signalling interval. The joint design in the first instance results in superior performance but with high complexity. The second instance is a special case of the first one and can be parameterized using a significantly smaller number of variables.

The joint design problem in both instances is nonconvex and locally optimal solutions are obtained using a GP-based monomial approximation technique. Numerical results show that the designs developed in both instances yield performance that is significantly better than that of their counterparts in which frequency-reuse is not allowed.

\section{APPENDIX A \\ THE GP STANDARD FORM AND MONOMIAL APPROXIMATION}

1) The GP Standard Form: For self-containment, in this appendix we will review the standard GP form. A GP optimization problem can be readily transformed to an efficiently solvable convex one. To provide the standard form of a GP, let $z \in \mathbb{R}^{n}$ be a vector of positive entries. A monomial in $z$ is defined to be a function of the form $c_{0} \prod_{i} z_{i}^{\alpha_{i}}$ and a posynomial in $z$ is defined to be a function of the form $\sum_{j=1}^{J} c_{j} \prod_{i=1}^{n} z_{i}^{\alpha_{i j}}$, where $c_{j}>0,\left\{\alpha_{i}\right\}$ and $\left\{\alpha_{i j}\right\}$, are arbitrary constants, $j=0,1, \ldots, J$, and $i=1, \ldots, n$. A standard GP [6], [7], [21] is an optimization of the form:

$$
\begin{aligned}
& \min _{z} f_{0}(z) \\
& \text { subject to } f_{i}(z) \leq 1, \quad i=1, \ldots, m \text {, } \\
& g_{i}(z)=1, \quad i=1, \ldots, p,
\end{aligned}
$$

where $\left\{f_{i}\right\}$ are posynomials and $\left\{g_{i}\right\}$ are monomials.

2) Monomial Approximation: A monomial approximation of a differentiable function $h(z) \geq 0$ near $z^{(0)}$ is given by its first order Taylor expansion in the logarithmic domain [6], [7]. Defining $\beta_{i}=\left.\frac{z_{i}^{(0)}}{h\left(z^{(0)}\right)} \frac{\partial h}{\partial z_{i}}\right|_{z=z^{(0)}}$, we have $\mathcal{M}(h(z))=$ $h\left(z^{(0)}\right) \prod_{i=1}^{n}\left(\frac{z_{i}}{z_{i}^{(0)}}\right)^{\beta_{i}}$, where $\mathcal{M}(\cdot)$ is the monomial approximation. This approximation will be used to provide local GP approximations in the neighbourhood of a given initial point.

\section{APPENDIX B \\ PROOF OF PROPOSITION 1}

For simplicity, we will begin by proving the second statement of Proposition 1 . In this proof, we will show that, by including redundant constraints, the log-barrier function of the problems in 30 and 22 can be cast in a self-concordant form, which has the following definition [21]:

Definition 1. A function $f: \mathbb{R}^{n} \rightarrow \mathbb{R}$ is said to be selfconcordant if, for all $x, v \in \mathbb{R}^{n}, s \in \mathbb{R}$ such that $x+s v$ is in the domain of $f$ and $\left|\frac{\partial^{3}}{\partial s^{3}} f(x+s v)\right| \leq 2 \frac{\partial^{2}}{\partial s^{2}} f(x+s v)^{3 / 2}$.

\section{A. Proof of the Second Statement of Proposition [1]}

To determine the complexity of solving the problem in (30), we begin by converting this problem into a convex one. Using standard exponential transformations, we write

$$
\begin{array}{lrl}
t_{n}^{(d)}=\exp \left(\ln (2) s_{n}^{(d)}\right), & n \in \mathcal{N} \backslash\{d\}, d \in \mathcal{D}, \\
r_{\ell k}^{(d)}=\exp \left(\ln (2) \frac{x_{\ell k}^{(d)}}{W}\right), & \ell \in \mathcal{L}, k \in \mathcal{K}, d \in \mathcal{D}, \\
y_{\ell k}=\exp \left(q_{\ell k}\right), & \ell \in \mathcal{L}, k \in \mathcal{K} .
\end{array}
$$

Substituting the variables in 30 with the ones in 33 and taking the logarithm of the obtained objective and constraints result in a convex optimization which can be solved efficiently using the IPM technique. To use this technique, a log-barrier function is synthesized from the objective and inequality constraints. The complexity analysis of the IPM 
technique is simplified when the log-barrier function is selfconcordant [21], cf., Definition 1]. The log-barrier function corresponding to the convex form of (30) can be written as

$$
\phi=-t \sum_{n} \sum_{d} w_{n}^{(d)} s_{n}^{(d)}+\psi,
$$

where $\psi$ represents the component of the log-barrier function associated with the inequality constraints in the convex form of (30). To examine whether $\phi$ is self-concordant, we note that the converted objective and the inequality constraints corresponding to (16), 17) and the relaxed versions of 24) and 25) are linear and therefore their corresponding components in the log-barrier function are self-concordant [21]. Hence it remains to consider the self-concordance for the constraints in 29. and (23). For simplicity, we write the posynomial constraint in (29) in the standard form in (32). After changing the variables and taking the logarithm of both sides, this constraint can be written in a general form as

$$
\log \left(\sum_{i} \exp \left(a_{i} \alpha_{i}+b_{i} \beta_{i}+c_{i}\right)\right) \leq 0,
$$

where $\left\{\alpha_{i}\right\},\left\{\beta_{i}\right\}$ are the optimization variables and $\left\{a_{i}\right\},\left\{b_{i}\right\},\left\{c_{i}\right\}$ are constants. The component corresponding to the constraint in 35 in the log-barrier function can now be expressed as

$$
-\log \left(-\log \sum_{i} \exp \left(a_{i} \alpha_{i}+b_{i} \beta_{i}+c_{i}\right)\right) .
$$

To ensure that 36 is self-concordant, we introduce auxiliary variables, $\lambda_{i}$, to bound the exponentially transformed variables in (35). Using these new variables, the constraint in 35 can be replaced with the following set of constraints [21]:

$$
\begin{aligned}
& \sum_{i} \lambda_{i} \leq 1, \\
& \lambda_{i} \geq 0, \\
& a_{i} \alpha_{i}+b_{i} \beta_{i}+c_{i}-\log \lambda_{i} \leq 0 .
\end{aligned}
$$

Now the associated log-barrier function of the constraints in (37) can be shown to be self-concordant, cf., [21, Example 9.8]. For the constraints in 23, we follow the steps analogous to the ones used with the constraints in 29. In particular, by introducing new auxiliary variables, we construct a self-concordant log-barrier function. Using this function, the complexity can be shown to be proportional to $m^{3.5}$, where $m$ is the number of inequality constraints. Hence, the complexity of solving (30) can be bounded by $\mathcal{O}\left((L K(3 L+2)+N+D(N-1))^{3.5}\right)$, which completes the proof of the second statement of Proposition 1

\section{B. Proof of the First Statement of Proposition 1}

The proof of the first statement of Proposition 1 follows from arguments similar to the one used in the proof of the second statement and is omitted for brevity. For the first statement, the number of inequality constraints can be readily verified to be $2 L K N+N+D(N-1)+2 K \sum_{i=1}^{I}\left(\begin{array}{c}L \\ i\end{array}\right)$, which yields the first statement of Proposition 1.

\section{REFERENCES}

[1] H. Yanikomeroglu, "Fixed and mobile relaying technologies for cellular networks," in 2nd Wksp. Apps and Svcs. in Wireless Networks, pp. 7581, July 2002.

[2] X. Bangnan, S. Hischke, and B. Walke, "The role of ad hoc networking in future wireless communications," in Proc. Int. Conf. Commun. Tech. (ICCT), pp. 1353-1358, Apr. 2003.

[3] S. Hayashi and Z.-Q. Luo, "Spectrum management for interferencelimited multiuser communication systems," IEEE Trans. Inf. Theory, vol. 55, pp. 1153-1175, Mar. 2009.

[4] H. Li and H. Liu, "An analysis of uplink OFDMA optimality," IEEE Trans. Wireless Commun., vol. 6, pp. 2972-2983, Aug. 2007.

[5] R. Rashtchi, R. H. Gohary, and H. Yanikomeroglu, "Routing, scheduling and power allocation in generic OFDMA wireless networks: Optimal design and efficiently computable bounds," IEEE Trans. Wireless Commun., vol. 13, pp. 2034-2046, Apr. 2014.

[6] M. Chiang, C. W. Tan, D. P. Palomar, D. O’Neil, and D. Julian, "Power control by geometric programming," IEEE Trans. Wireless Commun., vol. 6, pp. 2640-2650, July 2007.

[7] S. Boyd, S.-J. Kim, L. Vandenberghe, and A. Hassibi, "A tutorial on geometric programming," Optimization and Engineering, vol. 8, pp. 67127, Mar. 2007.

[8] B. R. Marks and G. P. Wright, "A general inner approximation algorithm for nonconvex mathematical programs," Operations Research, vol. 26, pp. 681-683, Aug. 1978.

[9] R. Rashtchi, R. Gohary, and H. Yanikomeroglu, "A cross-layer design for generic half-duplex interference-limited multicarrier networks," in IEEE Int. Wkshp Signal Processing Advances Wireless Commun. (SPAWC), June 2014.

[10] R. Rashtchi, R. H. Gohary, and H. Yanikomeroglu, "An efficient cross layer design in OFDMA-Based wireless networks with channel reuse," in Proc. IEEE Glob. Commun. Conf. (GLOBECOM), Dec. 2013.

[11] H. Inaltekin and S. V. Hanly, "Optimality of binary power control for the single cell uplink," IEEE Trans. Inf. Theory, vol. 58, pp. 6484-6498, Oct. 2012.

[12] K. Kim, Y. Han, and S.-L. Kim, "Joint subcarrier and power allocation in uplink OFDMA systems,” IEEE Commun. Lett., vol. 9, pp. 526-528, June 2005.

[13] C. Y. Ng and C. W. Sung, "Low complexity subcarrier and power allocation for utility maximization in uplink OFDMA systems," IEEE Trans. Wireless Commun., vol. 7, pp. 1667-1675, May 2008.

[14] J. Jang and K. B. Lee, "Transmit power adaptation for multiuser OFDM systems," IEEE J. Select. Areas Commun., vol. 21, pp. 171-178, Feb. 2003.

[15] J. Huang, V. G. Subramanian, R. Agrawal, and R. A. Berry, "Downlink scheduling and resource allocation for OFDM systems," IEEE Trans. Wireless Commun., vol. 8, pp. 288-296, Jan. 2009.

[16] L. Xiao, M. Johansson, and S. P. Boyd, "Simultaneous routing and resource allocation via dual decomposition," IEEE Trans. Commun., vol. 52, pp. 1136-1144, July 2004.

[17] K. Karakayali, J. Kang, M. Kodialam, and K. Balachandran, "Crosslayer optimization for OFDMA-based wireless mesh backhaul networks," in Proc. IEEE Wireless Commun. Ntwk Conf. (WCNC), pp. 276281, Mar. 2007.

[18] M. Johansson and L. Xiao, "Cross-layer optimization of wireless networks using nonlinear column generation," IEEE Trans. Wireless Commun., vol. 5, pp. 435-445, Feb. 2006.

[19] R. H. Gohary and T. J. Willink, "Joint routing and resource allocation via superposition coding for wireless data networks," IEEE Trans. Signal Processing, vol. 58, pp. 6387-6399, 2010.

[20] M. Charafeddine and A. Paulraj, "Sequential geometric programming for $2 \times 2$ interference channel power control," in Proc. IEEE Conf. Inf. Sci. Sys. , pp. 185-189, Mar. 2007.

[21] S. Boyd and L. Vandenberghe, Convex Optimization. Cambridge, UK: Cambridge University Press, 2004.

[22] Int. Telecommun. Union (ITU), Guidelines for Evaluation of Radio Interface Technologies for IMT-Advanced. ITU-R: TR M.21351, Dec. 2009. Available at: http://www.itu.int/pub/R-REP-M.2135-1-2009

[23] M. Grant and S. Boyd, CVX: Matlab Software for Disciplined Convex Programming, version 1.21, Jan. 2011. Available at: http://cvxr.com/cvx

[24] MOSEK Apps., The MOSEK Optimization Toolbox for Matlab Manual, Version 6.0, 2012. Available at: http://www.docs.mosek.com 